\title{
Vehicle Detection and Parameter Measurement using Smart Portable Sensor System
}

\author{
S.P. Jinturkar \\ PG Student, ENTC Dept \\ Sandip Institute of Technology and Research \\ Center Nasik, India
}

\author{
S.J. Pawar \\ Assistant Professor, ENTC Dept \\ Sandip Institute of Technology and Research \\ Center Nasik, India
}

\begin{abstract}
The study and observations of innovative portable roadside sensing system are done for the increasing number of losses in human live and also finance due to traffic accidents. The aim of this system to provide an efficient solution for automatic vehicle parameters measurement system. The wireless anisotropic magnetic sensor system embedded next to the roadway and examines the adjacent lane traffic measurements. Based on the calculation of time delay and distance between two longitudinally spaced AMR sensors. Categorization is done based on the magnetic length and average vertical magnetic height of the vehicle is calculated. The system can be used to reliably count the number of vehicles. The whole system is simple and conventionally used in real time measurements. This proposed system results will be significant supplement in management in traffic area and highways and automatic tollbooth.
\end{abstract}

\section{Keywords}

Anisotropic Sensors, portable roadside sensors, AMR sensors, real time measurement

\section{INTRODUCTION}

Traffic supervision and information systems depend on a set of sensors for calculating all parameters of traffic. Presently, magnetic loop sensing element and Visual sense-based video monitoring systems are often applied for counting the vehicles passing over them. Parameters as that vehicle classifications, and vehicle count, lane changes, can be computed. Likewise, cameras are difficult to install than other sensing systems like loop detectors. The current status is explained by obsolete data and, human operates manually at a particular street. The automated system is used for accurate design of pavements with axiomatic results in cost and quality. There is a need in large metropolitan cities to prevent from increasing number of accidents. This paper describes a roadside portable sensing system that can be embedded next to the road and can be used for speed estimation of vehicle, its counting, and vehicle classification. All these three parameters are considered necessary to study simultaneously for traffic management. The lane adjacent to the sensors is useful for the traffic parameter calculations. The sensor consists of magneto resistive devices that calculate the magnetic field. For the sensor estimation, signal processing algorithms on magnetic field around a car are used. Vehicle classification into predefined classes such as cars, trucks typically requires measuring the size or length of a vehicle, counting the number of axles of the vehicle [1].

A vehicle classification model based on feature extraction, accuracy rate is not $100 \%$ and can vary from $40 \%$ to $100 \%$. The vehicle classification and speed measurement is observed using multiple spatially separated magnetic sensors [6]. Many researchers study in this area. This paper starts by telling a summary of related piece of work, and then the study of conceptualization of system is presented, next session is with proposed results and discussion, and finally conclusion.

\section{RELATED WORK}

The traditional method proposed the vehicle classification information on a network-wide level [7], study of the feasibility of using inductive loop signatures is focused. Improvements in vehicle re-identification algorithms, roadway maintenance, emissions management of vehicles, roadway design, traffic modeling and simulation, traffic safety, and computerized toll collection caused by vehicle classification information. The compilation of a vehicle classification database is also an important resource for researchers in the areas of transportation planning and control.

The researcher studied to classify the TRV behavior on a moving measurement device is developed with the different ultrasonic sensor system [2].It is used in ITS \& VANET. To detection and counting of traffic a two-dimensional state method is used. But the limitation is only it will detect the other things passes from that sensors with vehicle. TRV system is established by combining two sensors to right side of each vehicle, assisted with a communication device for data transfer. Utilizing these two sensors continuously vehicle is detected, whether the some other vehicles overtaking it from the lower speed lane to its right side, after that the sensor system will noted the situation of the vehicle as a TRV behavior.

Only one sensor is not reliable to detect the passing vehicle because the reflected signal can be influenced by the target length, surface flatness, speed, height etc. Thus, the ultrasonic sensor system is designed to contain two parallel ultrasonic sensors, and the observed information of all sensors which act as a complementary factor for each other by using data processing techniques.

Researcher defined [9] the algorithms of complete system for vision-based detection and monocular image sequences classification of vehicles in traffic scenes. The algorithm contains the following stages are segmentation, Vehicle Identification, region tracking, restoration of vehicle parameters, vehicle classification vehicle tracking. In segmentation, the passing vehicles are separated from the background in the picture. After collection of all connected region, in the region tracking stage, using a spatial matching method, the region is tracked. To enable exact classification of the vehicles, the vehicle parameters need to be recovered from the 2-D projections of the vehicles. Vehicle Identification stage combined the tracked regions from the 
previous stage into vehicles. To enable tracking of vehicles, the system does tracking in the region level as well as the vehicle level. After vehicles have been detected and tracked, they are classified.

As regards the surveillance video camera, many works have been published. The work is proposed to use as a cost effective means to collect real-time data for each lane on roadways. An evaluation of three commercial camera based vehicle detection systems is presented in [4] under inimical weather conditions. The achievement of such systems deteriorates under unfavorable conditions, mostly under snow conditions in both daytime and nighttime. Video-based Vehicle Detection and Classification system can covered the digital video images or live video signal as input. First the users must know virtual loop locations, pixel-representation and a shadow sample to compose the system after that apply system for traffic data collection. Then background image is evoked from video input and updated regularly to acclimate to the environmental changes. After collecting the data, it observes the virtual loop for vehicle detection. A shadow of each detected vehicle is removed. Finally, a based on its pixelbased length and the LV threshold vehicle is classified into long vehicle (LV) bin or short vehicle (SV) bin.

The researcher newly utilized the progress in WSN [8] to develop a magnetic signature and length calculation based vehicle are classified with binary proximity magnetic sensor networks and the accuracy of vehicle surveillance is enhanced due to intelligent neuron classifier. In this methodology, to measure the magnetic field distortion, we use the low cost and high sensitive magnetic sensors, when vehicle passed the sensors and perceive vehicle via an adaptive threshold. The vehicle length is calculated with the geometrical characteristics of the proximity sensor networks, and from an intelligent neural network classifier vehicle type detected.

Researcher also worked on the wireless anisotropic magnetic sensor system that does not require to be embedded in the roadway - the sensors are embedded next to the roadway and measure traffic parameters in the immediately adjacent lane. A methodology based on a magnetic field model is robust the system to the errors created by larger vehicles driving in the nonadjacent lane.

Recently, [1] researcher anticipated three parameters that is vehicle counting, classification and speed measurement. Speed estimation conducted by placing two magnetic sensors horizontally apart and taking the cross-correlation between the signals from the two sensors. The speed estimation method is verified by mounting a test vehicle with a GPS. In [1] paper researcher study the experiment was conducted at the Minnesota Road Research Facility (MnROAD) for vehicle speed estimation. Adjacent to the lane, sensors were deployed. (Global Positioning System)GPS18 LVC was placed on a test vehicle, and its data were captured using a laptop inside the vehicle. And there is a separate data collection system was used to capture the roadside sensor data. There is a estimation error between the GPS measurement and the sensor based speed was calculated using the simple difference in the detection times. Proposed results show a $2.5 \%$ error in speed estimates over the range of $5-27 \mathrm{~m} / \mathrm{s}(11-60 \mathrm{mi} / \mathrm{h})$. Vehicle classification is performed based on the magnetic length and average magnetic height of vehicles. Vehicle length is estimated by multiplying the detection time by the observed speed. The average vertical magnetic height is recognized using two magnetic sensors that are vertically spaced.
Researcher also noted that system is used to count the number of vehicle passing. The limitation is only the classification rate is poor. But there is no traffic disturbance due to sensor system installation. And also the system is portable.

\section{OVERVIEW OF DIFFERENT SENSOR SYSTEMS 3.1 Vehicle Detection Technology}

Vehicle sensor and detection system are classified into intrusive and non-intrusive sensors. Intrusive sensors are sensors which are typically embedded in holes on the road surface by burrowed under road surface, or mounting the sensors on the road or above the road. Now days the sensors are embedded next to the roadways. Due to divergence of sensors in the market, scale of implementation, environmental conditions, cost, system design these factors would have to be taken while selecting the type of sensor.

Inductive loops, pneumatic road tubes, Active infrared sensors, magnetometers, magneto resistive sensors, piezoelectric cables and weight-in-motion sensors are examples of intrusive sensors. During installation and maintenance on or in the road causes inconvenience to the drivers. Non-intrusive sensors conclude the microwave radar, passive acoustic array sensors passive infrared sensors, RFID, ultrasonic and video image processing, etc. These classes of sensors overcome the disadvantages of intrusive sensor system of installation problem.

The different types of sensors used for vehicle detection and parameter measurements are discuss below:

\subsubsection{Active Infrared Sensors}

The sensor emitted the infrared energy and detecting the reflected energy. Multiple lane operations can be conducted by utilizing the active infrared sensors. An absolute measurement of vehicle position and class, many beams are transmitted from the sensor. The limitation is only its sensitivity towards environmental conditions which affects the operation of the sensor system.

\subsubsection{Inductive Loop Detector}

Inductive loop detectors (ILDs) are a widespread technology by many transportation agencies for vehicle detection and measurement of traffic flow rates. Individual vehicle speed is not measured by single inductive loop.

\subsubsection{Magnetometer}

Fluxgate magnetometer works by detecting magnetic anomaly in earth's magnetic field. These sensor system provides benefits of weather conditions like fog, snow rain etc. These sensors are more accurate and also not disturbed the traffic. The main disadvantages of using magnetometer are the small detection zones in some model which requires more than one unit are required for full lane detection and also proximity required for absolute detection.

\subsubsection{Magnetoresistive Sensor}

Lenz and Edelstein encompasses the Magnetoresistive sensors as Anisotropic Magnetoresistance Sensors (AMR) , Giant Magnetoresistance Sensors(GMR), Magnetic tunnel Junction sensors, Ballistic and Extraordinary Magnetoresistance that are simply energized by giving constant current. These sensors are small in size and light in weight which makes it versatile in placement. Other advantages are the low cost and 
wide range of temperature; it has been widely used for vehicle detection.

\subsubsection{Piezoelectric Sensors}

Piezoelectric sensors are form by the material which converts kinetic energy to electrical energy when subjected to vibrations. Based on weight it will provide more accurate data of vehicle speed and class of vehicle. To use multiple detectors to tackle the location, this is the advantage of these sensors. And it also has high sensitive to temperature.

\subsubsection{Pneumatic Road Tube}

Pneumatic road tube vehicle identify through air pressure created which closes the switch, create a signals when vehicle passed or stop over the tube. While it offers a low cost solution as well as quick installation and easy to maintain. Pneumatic road sensors are temperature sensitive. In addition, the tubes are prone to vandalism.

\subsubsection{Weight-In-Motion Sensors}

WIM sensors recognized the weight of the weight of vehicle. Whatever data observed is useful for highway planner, designers and law enforcement agencies. There are mainly four methods are available bending plate, piezoelectric, load cell and capacitance mat. Each sensor has its own benefits and also limitations. While bending plate WIM is more accurate and costly compared to piezoelectric sensor system, it is less accurate than load cell sensors and considered as cheaper.

\subsubsection{Acoustic Sensors}

Sensor system recognized vehicle through acoustic energy or audible sounds generated the vehicle by microphones installed for vehicle detection.

\subsubsection{RFID}

RFID having three units: transceiver, transponder, antenna and used for vehicle detection. The transceiver sends and read the information from transponder which contains coded data by antenna. For low cost installation and maintenance as well as high speed RFID is precise.

\subsubsection{Ultrasonic Sensors}

The sensors transmit the pulse waveforms between 25 to 50 $\mathrm{kHz}$ to the road by detecting transmitted energy which are reflected back the sensors. The reflected ultrasonic energies are analyzed to take data in roadside controller. It will do multiple lane detection with easy to install. Temperature change and extreme air turbulence affects the performance of sensor.

\section{SIGNAL STRENGTH AND SENSING SYSTEM CONFIGURATION}

An AMR sensor has a silicon chip and a thick layer of piezoresistive nickel-iron. The change the resistance of this layer caused due to presence of an automobile in close range breaks in the magnetic field. The Honeywell HMC5883 consists of surface-mount \& multi-chip module which are generally designed for low-field magnetic sensing for digital applications such as inexpensive compassing and magnetometry.

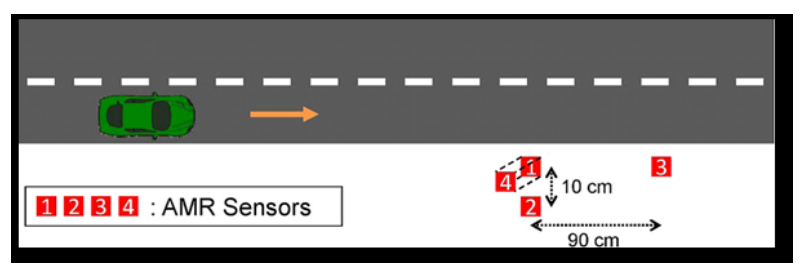

Fig 1: Sensor configuration for data compilation [1]

Fig. 1 represent the configuration of the sensing system, which having the four three-axis AMR sensors embedded on the side of the road. The main objectives of the project are: Automatic vehicle count detection, automatic height detection of the vehicle (whether high or low), automatic speed measurement display on LCD, zigbee based wireless data monitoring on PC Hyper terminal window. Sensors 1 and 2 are horizontally spaced from each other. Sensor 3 is placed longitudinally downstream from sensor 1 . Sensor 4 is placed above sensor 1 . Sensors 1 and 2 are together used to obtain a rough result of the sideway location of the vehicle and make the sensing system robust to the traffic in the nonadjacent lane.

\section{PROPOSED SYSTEM}

The aim of this project is to provide an efficient solution for automatic system for vehicle counting, classification, and speed measurement. The project makes use of a microcontroller, which acts as a central controlling unit. The system consists of two sections (i) sensor section: consists of three AMR sensors, LCD, zigbee interfaced to the PIC microcontroller. (ii) Monitoring section: consists of Zigbee, USB to TLL, and Interfacing to PC.

The PIC microcontroller is capable of communicating with the all modules i.e. input and output. In this system we are using three sensors to estimate vehicle counting, classification and speed.

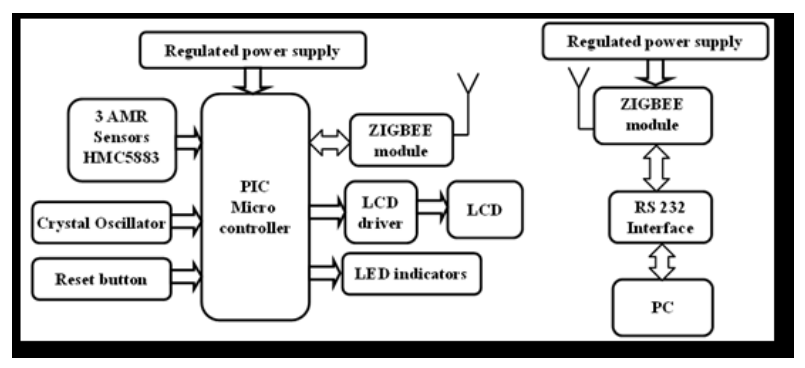

Fig 2: Block diagram of system

\subsection{Vehicle Detection and Counting}

From the previous section II the signals will record from the sensors embedded adjacent to the road are more uniform compared to the sensors are on the road. This behavior makes the detection more reliable. In particular, the signals measured along the $\mathrm{z}$-axis have very same patterns for a large variety of vehicles. Hence, magnetic readings of the $\mathrm{z}$-axis of sensor 3 are used for detecting and counting the passing vehicles in the adjacent lane.

\subsection{Speed Estimation}

The system measure the speed of vehicle using two sensors placed horizontally i.e. sensor $1 \& 2$. For setting speed limits and timing traffic signals transportation agencies use estimated speed information [11]. In the literature, there are some proposed methods for speed measurement using a single 
magnetic sensor [10]; however, those methods provide only an estimate of the speed over a number of passing vehicles. To measure each vehicle speed, two longitudinally spaced sensors are required. For measuring speed with two magnetic sensors is based on the detection times of the two sensors. If the time of detection vehicle for sensors "a" and "b" are ta, ON, ta, OFF, tb, ON, and tb, OFF, speed of vehicle can be calculated as:

$$
v=\frac{d a-b}{\left(\left(t_{b}, O N-t_{a}, O N\right)+\left(t_{b}, O F F-t_{a}, O F F\right)\right) / 2}
$$

Where, $\mathrm{da}-\mathrm{b}$ is the distance between sensors $\mathrm{a}$ and $\mathrm{b}$.

\subsection{Vehicle Classification}

Vehicle classification having various applications including traffic control, road maintenance and management, emissions evaluation, roadway design, multimode traffic model development, traffic signal design, and toll systems development as noted in paper [1]. The proposed system with results in literature for vehicle classification based on inductive loops and vision systems. But the main drawbacks of using inductive loops are the high cost, the long installation process, and the intrusive nature of sensor installation. The limitations to the systems based on vision are the high sensitivity to weather conditions, as mentioned in section II. The benefit of using magnetic sensors for vehicle classification and detection is that they are less expensive, adoptable to weather conditions, and easier to install and this system is potable, so we can placed adjacent to the road, and therefore, it is not necessary to disturbed the traffic.

\section{PROPOSED RESULTS AND DISCUSSION}

Vehicle counting, classification, \& speed estimation using portable sensor system will be proposed by reducing $20 \%$ error from previous system. The system will deal with 50 vehicles and $99 \%$ of accuracy in the result. The proposed system overcomes the traffic problem and works in all weather condition significantly. Especially in rainy season these system will work as it is. Due to easy installation of sensor system this technology is the best solution for the traffic management and construction of highways. The following fig. 3 shows the estimation of vehicle classes with measurement and examples of each [20].
Table 1: Parameter of vehicles

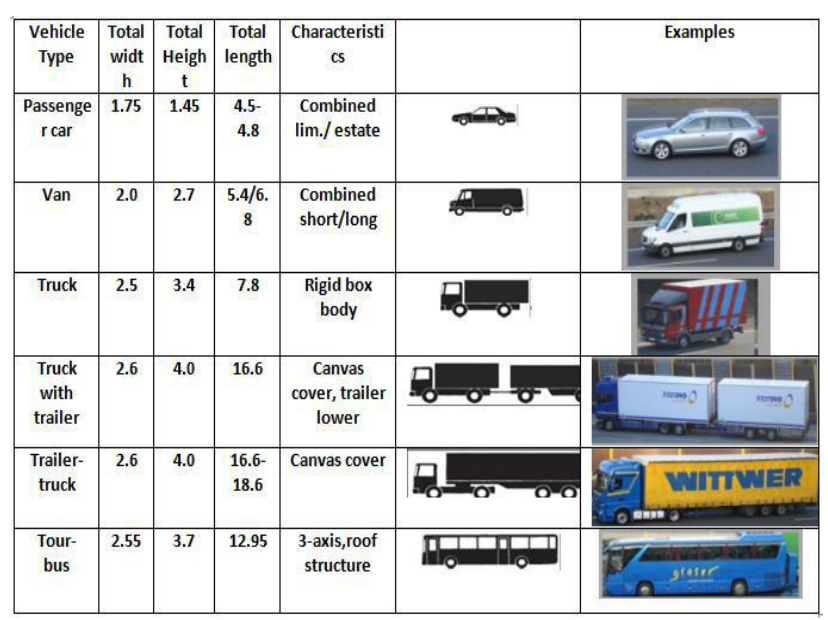

From the above table, the classification is planned using this system is more advantageous than other system.

\section{CONCLUSION}

To Vehicle detection and parameter measurements this system is useful. From the above, a portable and low-cost sensing system based on magnetic sensors system is more suitable than others. This proposed system adjacent to road can be used for various parameter measurement. These measurements are enabled using three separated magnetic sensors. The magnetic sensors are more versatile for all types of weather conditions. So, this proposed system results will be significant supplement in automatic Tollbooth, management in traffic area and highways.

\section{REFERENCES}

[1] Saber Taghvaeeyan and Rajesh Rajamani, February 2014, Portable Roadside Sensors for Vehicle Counting, Classification, and Speed Measurement, IEEE TRANSACTIONS.

[2] Jun Liu , Jiuqiang Han , Hongqiang Lv And Bing Li , An Ultrasonic Sensor System Based on a TwoDimensional State Method for Highway Vehicle Violation Detection Applications, ISSN 1424-8220

[3] D. P. Mishra, G. M. Asutkar, October 2013 .Vehicle Detection and Classification Using Wireless Sensor Network. International Journal of Advanced Research in Electrical, Electronics and Instrumentation Engineering.

[4] Guohui Zhang, Ryan P. Avery, TRB 2007 .A Videobased Vehicle Detection and Classification System for Real-time Traffic Data Collection Using Uncalibrated Video Cameras, Annual Meeting CD-ROM .

[5] Susmita A. Meshram, A.V. Malviya , November 2013. Traffic Surveillance by Counting and Classification of Vehicles from Video using Image Processing , International Journal of Advance Research in Computer Science and Management Studies.

[6] A.S. Lavanya, S.Srivani , August-2015. Real Time Portable Vehicle Counting and Speed Measurement Using Arm11 Processor, International Journal \& Magazine of Engineering, Technology, Management and Research. 
[7] Carlos, Sun, 2000,An Investigation in the Use of Inductive Loop Signatures for Vehicle Classification, Research Reports.

[8] Wei Zhang, Guo-Zhen Tan, Hui-Min Shi And Ming-Wen Lin, 2010.A Distributed Threshold Algorithm for Vehicle Classification Based On Binary Proximity Sensors And Intelligent Neuron Classifier. Journal Of Information Science And Engineering.

[9] Surendra Gupte, Osama Masoud, Robert F. K. Martin, and Nikolaos P. Papanikolopoulos. March 2002. Detection and Classification of Vehicles. IEEE TRANSACTIONS

[10] Cheung, Sing Yiu Coleri, Sinem Dundar, Baris Ganesh, Sumitra Tan, Chin-Woo Varaiya, Pravin,09-01-2004. Traffic Measurement and Vehicle Classification with a Single Magnetic Sensor, Working Papers.

[11] Y.-K. Ki and D.-K. Baik, Jul. 2006. Model for accurate speed measurement using double-loop detectors, IEEE Trans. Veh. Technol.

[12] Amine Haoui, Robert Kavaler, Pravin Varaiya,2007. Wireless magnetic sensors for traffic surveillance.Transportation Research Part C.

[13] Xiaotian Sun, March 31, 2006. A Set Of New Traffic Responsive Rampmetering Algorithms And Microscopic
Simulation Results, Transportation Research Record Journal Of The Transportation Research Board.

[14] Yao He,Yuchuan Du, Lijun Sun, August 1-3, 2012. Vehicle Classification Method Based on Single-Point Magnetic Sensor, 8th International Conference on Traffic and Transportation Studies Changsha, China.

[15] Guillaume Leduc, Road Traffic Data: Collection Methods and Applications, Working Papers on Energy, Transport and Climate Change N.1

[16] Wei Huang, 2014,Wireless Vehicle Detection Node based on Tunnelling Magneto Resistance Sensor, Computer Modelling \& New Technologies

[17] Pasquale Daponte, Luca De Vito, Francesco Picariello,Sergio Rapuano, Wireless Sensor network for Traffic safety.

[18] Jose Pelegri-Sebastia, Jorge Albe.ola ,2002. Vehicle Detection And Car Speed Monitoring System using GMR Magnetic Sensors, http://Www.Researchgate.Net/Publication/4005354

[19] Michael J. Caruso, Lucky S. Withanawasam, Vehicle Detection and Compass Applications using AMR Magnetic Sensors, www.ssec.honeywell.com

[20] www.ifh.uni-karlesruhe.de 